\title{
Juvenile Nephropathy in a Boxer Dog Resembling the Human Nephronophthisis- Medullary Cystic Kidney Disease Complex
}

\author{
Angelo BASILE ${ }^{1)}$, Andrea ONETTI-MUDA ${ }^{2)}$, Konstantinos GIANNAKAKIS ${ }^{3)}$, Tullio FARAGGIANA ${ }^{3)}$ and \\ Luca ARESU ${ }^{4)}$ \\ ${ }^{1)}$ Centro Nefrologico Veterinario, Via Cifali, 80-95123 Catania, ${ }^{2)}$ Department of Pathology, Campus Biomedico University, Via Alvaro \\ del Pontillo, 21-00128 Rome, ${ }^{3)}$ Department of Radiology, Oncology and Pathology, "Sapienza" University of Rome, Viale \\ dell'Università, 30-00185 Rome and ${ }^{4}$ Department of Public Health, Comparative Pathology and Veterinary Hygiene, Faculty of \\ Veterinary Medicine, University of Padua, Viale dell'Università, 16-35020 Agripolis,Padua, Italy
}

(Received 14 December 2010/Accepted 28 July 2011/Published online in J-STAGE 11 August 2011)

ABSTRACT. A juvenile nephropathy in a 4-year-old male Boxer dog, closely resembling the Nephronophthisis (NPHP)-Medullary Cystic Kidney Disease Complex (MCKD) in humans is described. Gross examination of the kidneys revealed several multiple cysts at the corticomedullary junction and in the medulla. Histological examination was characterized by a widespread tubular atrophy and dilatation, with a marked thickening of the tubular basement membrane, interstitial lymphocytic infiltration and fibrosis. Ultrastructural studies revealed dilated tubules with irregular basement membrane thickening and splitting. Lectin histochemistry investigation revealed that the cysts originated in the distal convoluted tubule and collecting duct. Having excluded all other known cystic diseases of the kidney, and based on the lectin histochemistry results, the macroscopic and histological findings of our case are highly compatible with a diagnosis of the NPHP-MCKD complex. To our knowledge, this is the first report describing this particular lesion. KEY WORDS: canine, cystic disease of the kidney, juvenile nephropathy, kidney, nephronophthisis.

J. Vet. Med. Sci. 73(12): 1669-1675, 2011

Cystic diseases of the kidney are a heterogeneous group of hereditary, acquired and developmental disorders characterized by the presence of multiple cysts in the kidneys [18]. A number of renal pathologies with a cystic component have been described in dogs including: Polycystic Kidney Disease (PKD) [25, 27, 32-34]; juvenile nephropathy (JN) [5, $26,35]$; renal dysplasia [12, 28, 30, 31, 36, 42]; medullary sponge kidney [2]; glomerulocystic kidney disease (GCKD)[4, 38, 46]; primary renal neoplasia such as hereditary multifocal renal cystadenocarcinomas and nodular dermatofibrosis (RCND) $33,19,20,29,48]$; renal cystadenoma [23], and acquired cysts [37]. PKD is a genetic disorder characterized by bilateral renal cysts of varying size and number distributed uniformly in the cortex and medulla [32]. The disease has been documented in Bull Terriers [32-34], Cairn Terriers [27] and West Highland White Terriers (WHWT) [25]. In Bull Terriers, PKD is inherited in an autosomal dominant manner, similar to human autosomal dominant polycystic kidney disease (ADPKD), whereas in the other two breeds pedigree analysis suggested an autosomal recessive mode of inheritance. Concurrent hereditary nephritis has been observed in Bull Terriers, whereas hepatic cysts characterize the disease in West Highland White and Cairn Terriers. JN refers to a variety of renal morphological pictures characterized by a disorganized nephrogenesis without the typical aspects of renal dysplasia and primary renal inflammation $[24,36]$, which induces chronic renal failure in young and middle-aged dogs. No gender predisposition has been reported. When a familial basis has been

\footnotetext{
* Correspondence to: Basile, A., Centro Nefrologico Veterinario, Via Cifali, 80-95123, Catania, Italy.

e-mail: angelofrancescobasile@gmail.com
}

detected, the condition is termed familial nephropathy [5, 35]. Small renal cysts ( $1 \mathrm{~mm}$ diameter) in the renal cortex are described [35] as a secondary degenerative change into interstitial fibrosis [5]. JN has been described in many different pure canine breeds $[35,39]$, boxer dogs, in particular, have been reported as being affected by either renal dysplasia [12] or JN [5, 15, 21, 35]. Renal dysplasia may be defined as a disorganized development of the renal parenchyma that is caused by the defective interaction of the ureteric bud and the metanephric blastema [36]. Renal dysplasia has been reported to occur infrequently in a large number of canine breeds $[1,12,14,28,30,31,42]$. The disease may be either inherited or acquired and two possible clinical manifestations [1] could be observed: a benign form in animals that are clinically well but with polyuria and polydipsia, and a more aggressive form in which polyuria and polydipsia are associated at a very early stage to signs of uraemia. Medullary sponge kidney is a rare, benign cystic disease affecting adults [18] and has been described in Shih Tzu dogs [2]. Cysts arise in the medullary collecting ducts and occupy the medulla of both kidneys like a sponge. GCKD is a condition characterized by a cystic dilatation of more than $5 \%$ of Bowman's spaces. It is described in human beings as either a primary, isolated form or in association with renal dysplasia and other malformations or acquired diseases. As a primary disorder, sporadic cases have been reported in canine breeds such as the Shiba [46], Belgian Malinoise [38] and Collie [4] breeds. Nothing is known about the pathogenesis and aetiology of canine primary GCKD. Moreover, it represents a secondary lesion in dogs affected by renal dysplasia, $\mathrm{JN}$, hereditary nephritis and progressive familial juvenile glomerulopathies (JGN) [17]. 
RCND is a naturally occurring, inherited cancer syndrome typically found in sporadic cases in German Shepherd [20] and Boxer dogs [48]. The disease is characterized by bilateral, multifocal tumours in the kidneys associated with nodular dermatofibrosis in both sexes [29] and multiple uterine leiomyomas in bitches [19, 20]. An autosomal dominant mode of inheritance has been postulated on the basis of pedigree analysis. The putative genetic defect has been located on exon 7 of a gene on chromosome 5 [19]. Interestingly, some genetic similarities are observed between RCND and human Birt-Hogg-Dubè syndrome [19]. Renal cystadenomas have been described in German shepherd, Golden Retriever and mixed-breed dogs [23, 48]. Multiple acquired renal cysts can develop in pre-existing normal nephrons and collecting duct systems in the course of many different conditions such as renal infection, renal neoplasia, tubule-interstitial nephritis and any chronic progressive renal disease [22]. The mechanism of cystogenesis is not completely understood and cysts can form without tubular obstruction.

Human "Nephronophthisis-Medullary Cystic Kidney Disease complex" (NPHP-MCKD) [10] is a group of human cystic diseases of the kidneys, defined as hereditary cystic diseases with interstitial nephritis [47]. Nephronophthisis (literally 'the disappearance of nephrons') was first described by Smith and Graham in 1945 and by Fanconi, who introduced the term "familial juvenile nephronophthisis" [45]. NPHP and MCKD share some clinical (polyuria, polydipsia and renal failure) and morphologic features (corticomedullary cysts in kidneys) but are clearly distinct as regards numerous other aspects, the first being onset of renal failure. NPHP is one of the main genetic causes of renal failure in children and young adults, while with MCKD renal failure occurs in adulthood. The second aspect concerns genetic transmission. NPHP is an autosomal recessive disorder, while MCKD is transmitted in an autosomal dominant pattern. Thirdly, NPHP is associated with extra-renal manifestations in $10-15 \%$ of patients [44], the most frequent disease being retinal degeneration. In the course of MCKD there is no extra-renal involvement other than hyperuricemia and gout [11]. The final aspect regards molecular and genetic pathogenesis. To date, mutations in twelve genes have been recognized to cause the different forms of NPHP[13]. All of these genes encode a group of proteins termed "nephrocystins", which have multiple cellular locations at the level of the collecting duct segment and are involved in different functions of this tract of the nephron, such as the primary cilia physiology, planar cell polarity, cell-cell contact and cell-matrix adhesion signaling, as well as cell division [13, 47]. Mutant nephrocystins can compromise epithelial integrity, in terms of growth, orientation, maintenance and repair, leading to tubular membrane disruption and induction of cystogenesis, particularly at the corticomedullary junction, where there is normally an abundant expression of nephrocystins [41]. The mutant nephrocystins can also alter the expression of the extra-cellular matrix causing interstitial flogosis and progressive intersti- tial and periglomerular fibrosis. The urine-concentrating defect and associated polyuria are associated with the defective primary cilia function, which depends on their role as mechanosensors sensing urinary flow [16]. Meanwhile, the localization of nephrocystins in the primary cilia, centrosomes and microtubule-based structures accounts for other extra-renal lesions, such as photoreceptor cilia in retinitis pigmentosa. MCKD is an autosomal dominant disorder, in which the mutated gene encodes uromodulin, also known as Tamm-Horsfall protein [47]. At present, the underlying mechanisms by which uromodulin is involved both in the tubular functional defect and in the tubular pathological lesions are not known.

From a clinical point of view, both the diseases are characterized by a decreased urine-concentrating capacity, with polyuria and polydipsia and progressive renal failure [11].

The present report describes the case of a male middleaged Boxer dog with renal failure in which the underlining disease was diagnosed as $\mathrm{JN}$ with a peculiar cystic expression, a combination not previously reported in dogs, which shares some clinical and histological features with NPHPMCKD.

A 4-year-old male Boxer was presented with a 5-day history of persistent vomiting. The clinical history excluded any exposure to toxic substances. The owner also reported polyuria, polydipsia and weight loss over a period of 8 months. On physical examination, the dog was underweight, body temperature and blood pressure were normal. No nodular lesions were detected on the skin of the dog. Serum biochemical tests and haematology revealed markedly elevated serum creatinine $(12 \mathrm{mg} / \mathrm{d} l$, normal range $0.5-$ $1.2 \mathrm{mg} / \mathrm{d} l)$, serum urea nitrogen $(276 \mathrm{mg} / \mathrm{d} l$, normal range $15-30 \mathrm{mg} / \mathrm{d} l)$, hyperphosphatemia $(9.8 \mathrm{mg} / \mathrm{d} l$, normal range $3.5-5.5 \mathrm{mg} / \mathrm{d} l)$ and non-regenerative, normochromic, normocytic anaemia $\left(\mathrm{RBC} 2.34 \times 10^{12} / l\right.$, normal range 5.5-7.9 $\times 10^{12} / l$ ). Urinalysis indicated isosthenuria (specific gravity 1.012), proteinuria (dipstick 4+), microscopic haematuria (10 RBC $\times$ high power field), leucocyturia (20-30 white blood cells $\times$ high power field) without bacteriuria. The urine protein:creatinine (UPC) ratio was high at 7.7 (reference range $<0.5$ ). An aerobic bacterial culture of the urine yielded no growth. Ultrasonographic examination showed hypoecogenic rounded formations in both kidneys, mainly along the corticomedullary junction. Proximal ureters showed absence of dilatation. No others lesions were detected in the remaining abdominal organs. A clinical diagnosis of acute-on-chronic renal failure associated with a bilateral cystic nephropathy was formulated. Based on the IRIS system [37], the dog was classified as stage 4, P, AP0. Due to the poor prognosis, the dog was humanely destroyed. Macroscopic examination at necropsy revealed pathological changes limited exclusively to the kidneys, which however did not appear to be significantly enlarged.

The kidneys were fixed in 10\% neutral buffered formalin and sectioned mid-sagittally; the long axis of the fixed kidneys was around $7 \mathrm{~cm}$ and the width was about $3.5 \mathrm{~cm}$. On the cut surface, the renal cortex $(0.5 \mathrm{~cm}$ in width) was char- 


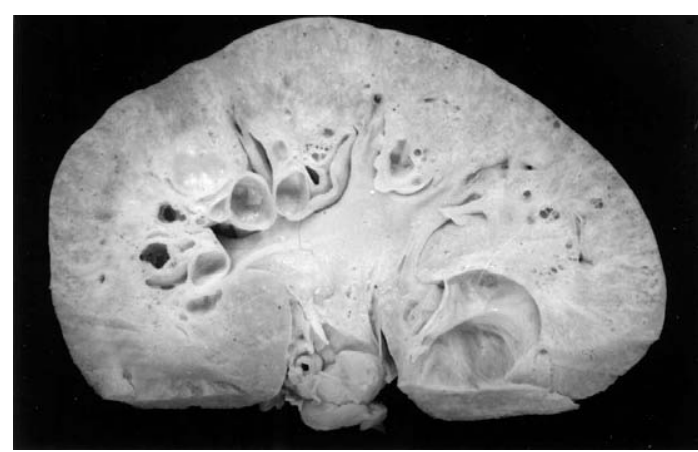

Fig. 1. Left kidney: sagittal section view. Numerous large and small cysts occupy the corticomedullary junction and the medulla.

acterized by the presence of small cysts, ranging from 1 to 3 $\mathrm{mm}$ in diameter. The medulla and the corticomedullary junction revealed several cysts, from 4 to $7 \mathrm{~mm}$ in diameter (Fig. 1), containing a colourless to reddish fluid. In addition, a larger cyst $(2.2 \mathrm{~cm})$ occupied the caudal pole of the left kidney, extending from the capsule to the inner medulla. Both the pelvis and ureters appeared normal. Sampling was performed in selected areas of the cortex, medulla and corticomedullary junction area of both kidneys; renal tissue specimens were processed by routine methods for histological examination and stained with haematoxylin and eosin (HE), Periodic Acid-Schiff (PAS) and Masson Trichrome stains. Identification of the nephron segment from which the cysts originated was performed by lectin histochemistry using peroxidase-labelled lectins from Arachis Hypogaea (Peanut lectin, PNA) and Lotus tetragonolobus (Tetragonolobus purpureas, TP) (Sigma Chemical Company, Milan, Italy). PNA, a binding terminal non-reducing Dgalactose, is a marker for distal convoluted tubules (DCT) and collecting ducts (CD), while TP has a high affinity for $\alpha$-L-fucose, and stains the brush border of the proximal tubule [7, 40]. Type IV collagen was used to study the tubular basement membrane. Additional renal specimens were postfixed in $1 \%$ osmium tetroxide and embedded in Epon for ultrastructural examination. Thin sections (700 A) were stained with uranyl acetate and lead citrate and observed under a transmission electron microscope (Philips CM 10). The macroscopic and microscopic features are summarized in Table 1.

Under light microscopy, the tubular-interstitial compartment showed: a) tubular dilatation and atrophy with a marked disruption of the normal aspect of the tubular basement membrane (TBM) characterized by irregular thickening as well as attenuation; b) diffuse interstitial lymphocytic-plasmacellular infiltrate; c) interstitial fibrosis (Fig. 2). The glomerular compartment showed cystic dilatations of Bowman's space with marked atrophy of the tufts and pericapsular fibrosis (Fig. 3). The average density of the atrophic glomeruli was roughly $15 \%$ at high power magnification. Multiple cysts of varying diameters were found at the corticomedullary junction and in the medulla (Fig. 4). The cysts were lined by cubic or flattened epithelium lying on a thickened tubular basement membrane. The connective tissue, just beneath the urothelium of the renal pelvis, was diffusely infiltrated by lymphocytes, plasma cells and many neutrophils, the latter forming numerous microabscesses in the urothelium.

Lectin histochemistry showed that, the apical membrane of the epithelial cells lining the cysts was neatly labelled by PNA (Fig. 5a), and was consistently negative with TP (Fig. $5 b)$, identifying their origin from DCT and CD. Moreover, collagen IV immunostaining displayed a diffuse positivity along the tubular basement membrane, confirming the integrity of the collagen structure in this dog (Fig. 6). Ultrastructural examination revealed that the basement membrane of the dilated tubules was greatly thickened and laminated. Fibrillary collagen was also present, intermingled with the non-fibrillary amorphous and homogenous matrix (Fig. 7). The basement membrane of Bowman's capsule appeared normal. The morphological features, age, breed and clinical history of the animal were compatible with both congenital and acquired nephropathy, with cyst formation [5].

The differential diagnosis of our case included PKD, renal dysplasia, medullary sponge kidney, GCKD, RCND, acquired cyst disease and JN. PKD, either autosomal reces-

Table 1. Summary of macroscopic and microscopic findings in both kidneys

\begin{tabular}{lll}
\hline Macroscopic & Tubuli & $\begin{array}{l}\text { Corticomedullary and Medullary Cysts } \\
\text { Dilatation and Atrophy } \\
\text { Irregular thickening and attenuation of TBM } \\
\text { Cysts lined by flattened ephitelium } \\
\text { Lymphocytic-plasmacellular infiltrate }\end{array}$ \\
& Interstitium & $\begin{array}{l}\text { Fibrosis } \\
\text { Cystic dilatation of Bowman's space with atrophy } \\
\text { of glomerular tuft }\end{array}$ \\
& Pericapsular fibrosis \\
Ephitelium lining the cysts positive bounded by TP \\
(origin from DCT and CD) \\
Lamina densa thickened and laminated \\
Non-fibrillar amorphous matrix intermingled \\
between collagen fibers
\end{tabular}

Abbreviations: TBM tubular basement membrane; TP Tetragonolobus purpureas; DCT distal convoluted tubule; $\mathrm{CD}$ collecting duct. 


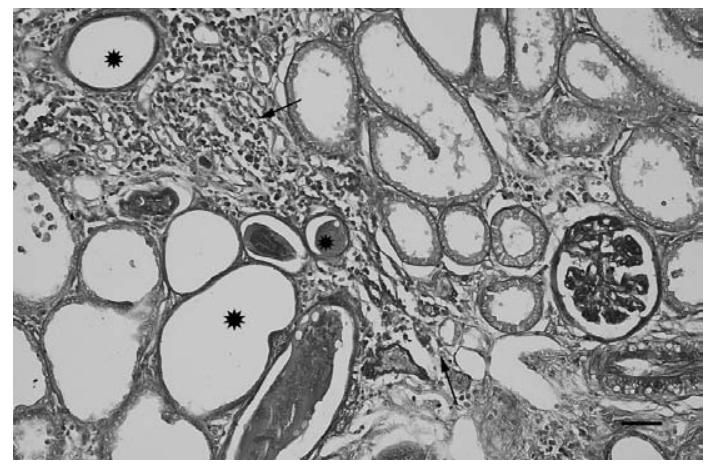

Fig. 2. Kidney: light microscopy shows atrophic, dilated tubules with irregular thickening of the basement membrane (asterisks) and areas of interstitial fibrosis and lymphocytic infiltrate (arrows) (PAS; $\times 100$. Bar: $100 \mu \mathrm{m}$ ).

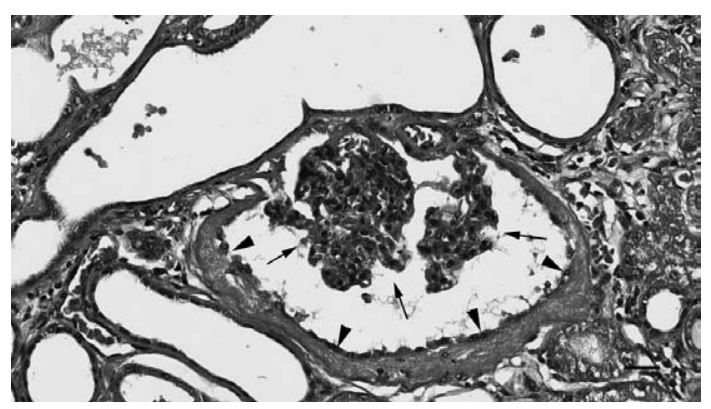

Fig. 3. Kidney: light microscopy showing cystic dilatations of Bowman's space with ischemic, atrophic glomerular tuft (arrows) and fibrotic Bowman's capsule (arrowheads) with pericapsular fibrosis (HE; $\times 400$. Bar: $50 \mu \mathrm{m})$.

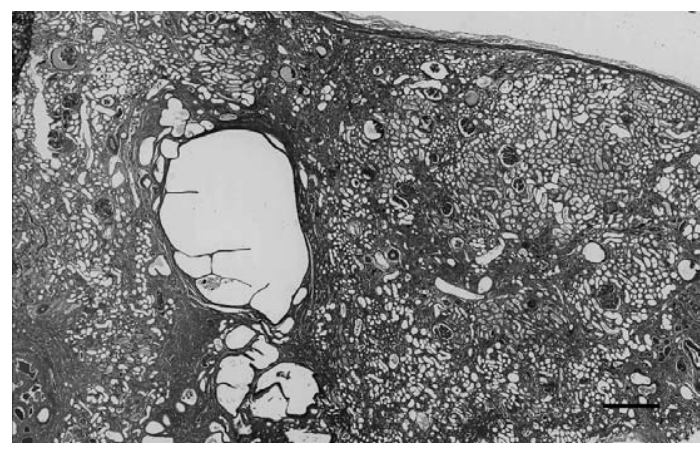

Fig. 4. Kidney: light microscopy shows cysts of different sizes at the corticomedullary junction lined by cuboidal or flattened epithelium (PAS; $\times 250$. Bar: $1 \mathrm{~mm}$ ).

sive or dominant, is characterized by cysts distributed uniformly over the entire organ and the kidneys are enlarged. [32]. The more recent papers [47] indicate that even if bilateral renal cysts are located at any point along the length of the nephron, at early stages, the majority of cysts derive from the distal nephron and the collecting duct, whereas the other segments of the nephron are involved in cystic dilata-

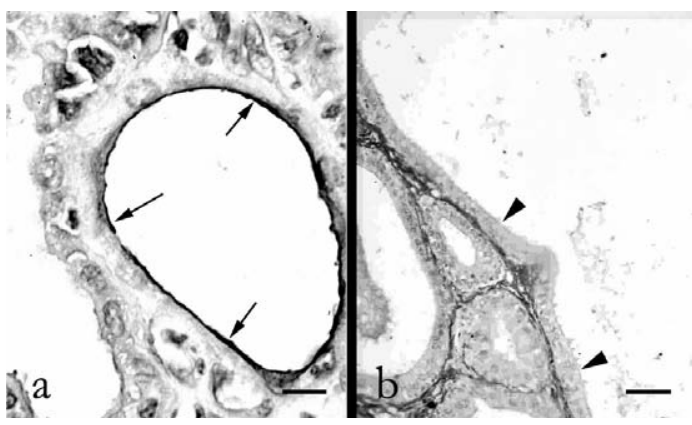

Fig. 5. Kidney. Lectin histochemistry: renal medulla. a) PNA lectin. The luminal surface of the epithelial lining of the dilated ducts shows positive staining (arrows), thus demonstrating its origin from distal ducts (PAPDAB; $\times 650$. Bar: $10 \mu \mathrm{m})$. b) TP lectin. The luminal surface of the cells lining the cysts is constantly negative (arrowheads) (PAP-DAB; $\times 400$. Bar: $50 \mu \mathrm{m}$ ).

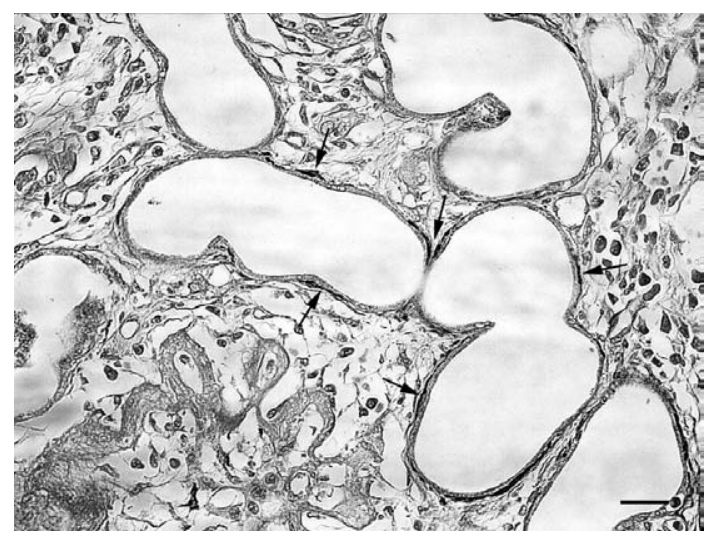

Fig. 6. Kidney. Immunostaining for collagen IV: diffuse intense positivity along the thickened basement membrane of cystic tubules (arrows) (PAP-DAB; $\times 200$. Bar: 75 $\mu \mathrm{m})$.

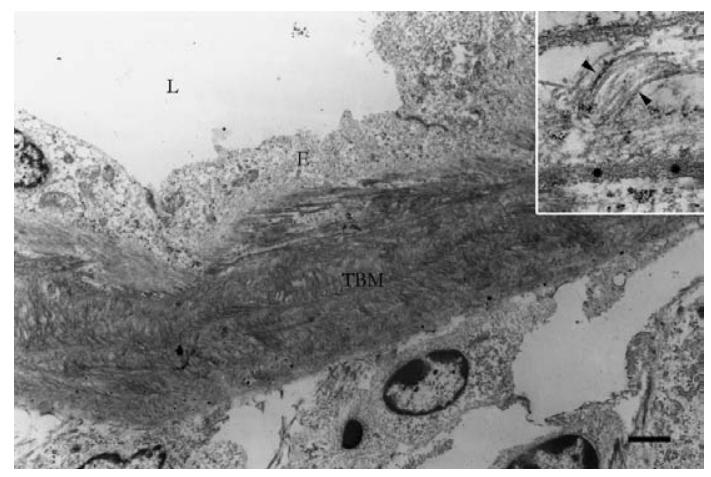

Fig. 7. Kidney. Ultrastructural appearance of the cyst wall. The basement membrane of the epithelial lining (TBM) is markedly thickened due to accumulation of fibrillary collagen (arrowheads) and amorphous, non-fibrillary matrix (astericks) (insert). (Uranyl acetate-lead citrate; $\times 10,000$. Bar: $1 \mu \mathrm{m})($ Insert: $\times 75,000)$. 
tion only at later stages of the disease.

Renal dysplasia has been reported in Boxer dogs [12, 21] and cyst formation is possible as a result of intra-renal obstruction due to interstitial fibrosis. This diagnosis was excluded by the absence of the typical morphologic features, i.e. the presence of metanephric ducts surrounded by primitive mesenchyme and fetal or immature glomeruli and tubules [36]. Medullary sponge kidney was excluded simply by the typical localization of the cysts in this rare disease. In the course of GCKD and JGN [17] minimal or small cysts are bilaterally and uniformly distributed throughout the cortex of normal-sized kidneys. The cysts originate from the dilatation of Bowman's space and proximal tubule with mild interstitial fibrosis and the condition is considered primary when the prominent features are the cystic dilatation of the urinary space and poor development of the glomerulus. A glomerulocystic component, however, can be associated with other cystic conditions such as PKD or secondary to interstitial fibrosis or tubular atrophy.

In the course of RCND both kidneys are usually enlarged due to the presence of numerous cortical cysts. The cysts are believed to originate from renal tubules occluded by hyperplastic-to-dysplastic and neoplastic epithelium. Small renal macroscopically-visible cysts could be observed in affected dogs as early as $6-8$ weeks of age [3]. This particular disease has been described in a Boxer dog [48] but in our case neither nodular dermatofibrosis nor neoplastic signs were seen. Nodular dermatofibrosis is considered a marker for RCND, even if it has been described either in the absence of RCND [9] or associated with renal cystadenomas in Golden Retrievers [23] and mixed-breed dogs [48]. Acquired cysts as a consequence of chronic renal failure could also be excluded. Cysts may have a similar distribution to that in PKD in the parenchyma of both kidneys. Unlike PKD, the kidneys tend to be either of normal size or slightly smaller [18]. Acquired cysts originate from proximal and distal tubules and are lined by cuboidal or flat epithelium. The main histopathological findings of all the JN cases reported in veterinary literature $[5,15,26,35,39]$ could be summarized in: pericapsular and interstitial fibrosis, interstitial round-cell infiltrate, tubular dilatation with thickening of the TBM and a marked dilatation of Bowman's space with atrophy of the glomerular tuft, without evidence of either fetal glomeruli or persistent primitive tubules. The presence of renal cysts were cited only in the ultrasonographic findings but neither their localization inside the kidney nor their detailed histopathologic description were given. Moreover, there is no ultrastructural or immunoistochemical description of JN. Our dog shares history, clinical signs and histopathological features with $\mathrm{JN}$. Unlike classic $\mathrm{JN}$, the gross anatomy and light microscopic pictures of the boxer dog reported in our paper are dominated by the presence of multiple cysts localized at the corticomedullary junction. This histological finding closely resembles the NPH-MCKD complex.

As far as the macroscopic pathology features are concerned, the NPH-MCKD complex is characterized by small- to-normal-sized kidneys with cysts typically localized at the corticomedullary junction of the kidney and in the renal medulla. Under light microscopy the histological features occur mainly in the tubular compartment and are characterized by disruption of the tubular basement membrane with irregular thickening and attenuation and later on, tubular atrophy with the development of cysts, occurring primarily at the corticomedullary junction [10]. At the interstitium, the signs consist of round cell infiltration and marked fibrosis. The only significant glomerular lesion involves periglomerular fibrosis in the early stages and secondary glomerulocystic degeneration with atrophy of the tufts, later [10]. Microdissection and histochemical studies have shown that the cysts arise from the distal convoluted and collecting tubules $[8,18,43]$. In particular, lectin histochemistry has demonstrated that cysts originate from the distal convoluted tubules and collecting ducts $[8,43]$. No abnormalities were found staining with antibodies to laminin and type IV collagen [6]. Electron microscopy showed dilated tubules with characteristic tubular basement membrane (TBM) changes $[6,18]$ : thickening, splitting and lamination, alternating with thin or absent segments.

In conclusion, both macroscopic appearance and microscopic findings, including light microscopy, lectin histochemistry, immunohistochemistry and an ultrastructural study of our dog are very similar to those observed in the human NPHP-MCKD complex. Unfortunately, we were unable to demonstrate a hereditary or familial condition in this case due to the absence of any data regarding the pedigree of the dog.

The onset of chronic renal failure in juvenile, adolescent or young adult Boxer dogs could have a pathological background in both renal dysplasia $[12,21]$ and $\mathrm{JN}[5,15,35]$ with some peculiar patterns of expression. Further investigation is required to verify whether NPHP might represent another potential cause.

ACKNOWLEDGMENTS. The authors are deeply grateful to Mr. Giancarlo Bolognesi, Mr. Lucio Virgilii and Mrs Fausta Fanella for technical assistance.

\section{REFERENCES}

1. Abraham, L. A., Beck, C. and Slocombe, R. F. 2003. Renal dysplasia and urinary tract infection in a bull Mastiff puppy. Aust. Vet. J. 81: 336-339.

2. Akihara, Y., Shimoyama, K., Ohya, A., Hirayama, K., Yoshida, K., Matsuda, M. and Taniyama, H. 2006. Medullary sponge kidney in a 10-year-old shih tzu dog. Vet. Pathol. 43: 10101013.

3. Bønsdorff, T. B., Jansen, J. H., Thomassen, R. F. and Lingaas, F. 2009. Loss of heterozygosity at the FLCN locus in early renal cystic lesions in dogs with renal cystadenocarcinomas and nodular dermatofibrosis. Mamm. Genome 20: 315-320.

4. Chalifoux, A., Phaneuf, J. B., Olivieri, M. and Gosselin, Y. 1982. Glomerular polycystic kidney disease in a dog (Blue Merle Collie). Can. Vet. J. 23: 365-368.

5. Chandler, M. L., Elwood, C., Murphy, K. F., Gajanayake, I. and Syme, H. M. 2007. Juvenile nephropathy in 37 boxer dogs. 
J. Small Anim. Pract. 48: 690-694.

6. Cohen, A. H. and Hoyer, J. R. 1986. Nephronophtisis. A primary tubular basement membrane defect. Lab. Invest. 55: 564572.

7. Faraggiana, T., Bernstein, J., Strauss, L. and Churg, J. 1985. Use of lectins in the study of histogenesis of renal cysts. Lab. Invest. 53: 575-579.

8. Faraggiana, T., Malchiodi, F., Prado, A. and Churg, J. 1982. Lectin-peroxidase conjugate reactivity in normall human kidney. J. Histochem. Cytochem. 30: 451-458.

9. Gardiner, D. W. and Spraker, T. R. 2008: Genaralized nodular dermatofibrosis in the absence of renal neoplasia in an Australian Cattle dog. Vet. Pathol. 45: 901-904.

10. Hildebrandt, F. and Omram, H. 2001. New insights: nephronophthisis-medullary cystic kidney disease. Pediatr. Nephrol. 16: $168-176$.

11. Hildebrandt, F. and Zhou, W. 2007. Nephronophthisis-associated ciliopathies. J. Am. Soc. Nephrol. 18: 1855-1871.

12. Hoppe, A. and Karlstam, E. 2000. Renal dysplasia in Boxers and Finnish harriers. J. Small Anim. Pract. 41: 422-426.

13. Hurd, T. W. and Hildebrandt, F. 2011. Mechanisms of nephronophthisis and related ciliopathies. Nephron. Exp. Nephrol. 118: e9-e14.

14. Kerlin, R. L. and Winkle, T. J. 1996. Renal dysplasia in Golden Retrievers. Vet. Pathol. 32: 327-329.

15. Kolbjørnsen, Ø., Heggelund, M. and Jansen, J. H. 2008. Endstage kidney disease probably due to reflux nephropathy with segmental hypoplasia (Ask-Upmark kidney) in young Boxer dogs in Norway. A retrospective study. Vet. Pathol. 45: 467474.

16. Krishnan, R., Eley, L. and Sayer, J. A. 2008. Urinary concentration defects and mechanisms underlying nephronophthisis. Kidney. Blood Press Res. 31:152-162.

17. Lavouè, R., van der Lugt, M. J., Day, M. J., Georges, M., Busoni, V., Merveille, A. C., Poujade, A. and Peeters, D. 2010. Progressive juvenile glomerulonephropathy in 16 related French Mastiff (Bordeaux) dogs. J. Vet. Intern. Med. 24: 314 322.

18. Liapis, H. and Winyard, P. 2007. Cystic diseases and developmental kidney defects. pp. 1273-1278. In: Heptinstall's Pathology of the KIDNEY, 6th ed. (Jennette, J. C., Olson, J. L., Scwhartz M. M. and Silva F. G. Eds.), Lippincott Williams \& Wilkins, Philadelphia.

19. Lingaas, F., Comstock, K. E., Kirkness, E. F., Sørensen, A., Aarskaug, T., Hitte, C., Nickerson, M. L., Moe, L, Schmidt, L. S., Thomas, R., Breen, M., Galibert, F., Zbar, B. and Ostrander, E. A. 2003. A mutation in the canine BHD gene is associated with hereditary multifocal renal cystadenocarcinomas and nodular dermatofibrosis in the German Shepherd dog. Hum. Mol. Genet. 12: 3043-3053.

20. Lium, B. and Moe, L. 1985. Hereditary multifocal renal cystadenocarcinomas and nodular dermatofibrosis in the German shepherd dog: macroscopic and histopathologic changes. Vet. Pathol. 22: 447-455.

21. Lucke, V. M., Kelly, D. F., Darke, P. G. and Gaskell, C. J. 1980. Chronic renal failure in young dog-possible renal dysplasia. J. Small Anim. Pract. 21: 169-181.

22. Lulich, J. P., Osborne, C. A. and Polzin, D. J. 1995.Cystic diseases of the kidney. pp. 460-470. In: Canine and Feline Nephrology and Urology (Osborne, C. A. and Finco, D. R. Eds.), Williams \& Wilkins, Baltimore.

23. Marks, S. I., Farman, C. A. and Peaston, A. 1993: Nodular dermatofibrosis and renal cystadenoma in a Golden Retriever. Vet.
Dermatol. 4: 133-137.

24. Maxie, M. G. 1993. The urinary system: Anomalies of development. pp. 459-468. In: Pathology of Domestic Animals, Vol. 2, 4th ed. (Jubb, K. V. F., Kennedy, P.C. and Palmer, N. eds.), Academic Press, New York.

25. McAloose, D., Casal, M., Patterson, D. F. and Dambach, D.M. 1998. Polycystic kidney and liver disease in two related west highland white terrier litters. Vet. Pathol. 35: 77-81.

26. McKay, L. W., Seguin, M. A., Ritchey, J. W. and Levy, J. K. 2004. Juvenile nephropathy in two related Pembroke welsh corgi puppies. J. Small Anim. Pract. 45: 568-571.

27. McKenna, S. C. and Carpenter, J .L. 1980. Polycystic disease of the kidney and liver in the Cairn Terrier. Vet. Pathol. 17: 436-442.

28. Miyamoto, T., Wakizaka, S., Matsuyama, S., Baba, E., Ohashi, F., Kuwamura, M., Yamate, J. and Kotani, T. 1997. A control of a Golden Retriever with renal dysplasia. J. Vet. Med. Sci. 59: 939-942.

29. Moe, L. and Lium, B. 1997. Hereditary multifocal renal cystadenocarcinomas and nodular dermatofibrosis in 51 German shepherd dogs. J. Small Anim. Pract. 38: 498-505.

30. Morita, T., Michimae, Y., Sawada, M., Uemura, T., Araki, A., Haruna, A. and Shimada, A. 2005. Renal dysplasia with unilateral renal agenesis in a dog. J. Comp. Pathol. 133: 64-67.

31. Ohara, K., Kobayashi, Y., Tsuchiya, N., Furuoka, H. and Matsui, T. 2001. Renal dysplasia in a Shih tzu dog in Japan. J. Vet. Med. Sci. 63: 1127-1130

32. O'Leary, C. A., Ghoddusi, M. and Huxtable, C. R. 2002. Renal pathology of polycystic kidney disease and concurrent hereditary nephritis in Bull Terriers. Aust. Vet. J. 80: 353-361.

33. O’Leary, C. A., Mackay, B. M., Malik, R., Edmondston, J.E., Robinson, W. F. and Huxtable, C. R. 1999. Polycystic kidney disease in bull terriers: an autosomal dominant inherited disorder. Aust. Vet. J. 77: 361-366.

34. O'Leary, C. A. and Turner, S. 2004. Chronic renal failure in an English bull terrier with polycystic kidney disease. J. Small Anim. Pract. 45: 563-567.

35. Peeters, D., Clercx, C., Michiels, L., Desmecht, D., Snaps, F., Henroteaux, M. and Day, M .J. 2000. Juvenile nephropathy in a Boxer, a Rottweiler, a Collie and an Irish Wolfhound. Aust. Vet. J. 78: 162-165.

36. Picut, C. A. and Lewis, R. M. 1987. Microscopic features of canine renal dysplasia. Vet. Pathol. 24: 156-163.

37. Polzin, D. J., Osborne, C. A. and Ross, S. 2005. Chronic kidney disease. pp. 1756-1785. In: Textbook of Veterinary Internal Medicine, 6th ed. (Ettinger S. J. and Feldman E. C. eds.), Elsevier Saunders, Philadelphia.

38. Ramos-Vara, J. A., Miller, M. A., Ojeda, J.L., Reid, R. R., Craft, D. and Watson, G. L. 2004. Glomerulocystic kidney disease in a Belgian Malinois dog: an ultrastructural, immunohistochemical, and lectin-binding study. Ultrastruct. Pathol. 28: 33-42.

39. Reilly, C. M., Munson, L. and Bell, J. S. 2007. Progressive juvenile nephropathy in Gordon Setters. In abstract combined 58th and 42nd ACVP and ACSVCP. Vet. Pathol. 44: 732-785.

40. Risdon, R. A. and Woolf, A. S. 1998. Developmental defects and cystic diseases of the kidney. pp. 19-20. In: Heptinstall's Pathology of the Kidney, 5th ed. (Jennette, J. C., Olson, J. L., Schwartz, M. M. and Silva, F. G. eds.), Lippincott-Raven, Philadelphia.

41. Saunier, S., Salomon, R. and Antignac, C. 2005. Nephronophthsis. Curr. Opin. Genet. Dev. 15: 324-331.

42. Schulze, C., Meyer, H. P., Blok, A. L., Schipper, K. and van 
den Ingh, T. S. 1998. Renal dysplasia in three young adult Dutch kookier dogs. Vet. Q. 20: 146-148.

43. Seshan, S. V., D’Agati, V. D., Appel, G. A. and Churg, J. 1999. Hereditary and primary tubulo-interstitial disorders. pp. 185187. In: Renal Disease : Classification and Atlas of Tubulointerstitial and Vascular Diseases (Seshan, S. V., D'Agati, V. D., Appel, G. A. and Churg, J. eds.), Williams \& Wilkins, Baltimore.

44. Simms, R. J., Hynes, A. M., Eley, L. and Sayer, J. A. 2011. Nephronophthisis: a genetically diverse ciliopathy. Int. J. Nephrol. 2011: 527137.

45. Simms, R. J., Eley, L. and Sayer, J. A. 2009. Nephronophthisis.
Eur. J. Hum. Genet. 17: 406-416.

46. Takahashi, M., Morita, T., Sawada, M., Uemura, T., Haruna, A. and Shimada, A. 2005. Glomerulocystic kidney in a domestic dog. J. Comp. Pathol. 133: 205-208.

47. Torres, V. E. and Grantham, J. J. 2008. Cystic diseases of the Kidney. pp. 1428-1462. In: Brenner \& Rector's The Kidney, 8 th ed. (Brenner, B. M. rd.), Elsevier Saunders, Philadelphia.

48. White, S. D., Rosychuk, R. A. W., Schultheiss, P. and Scott, K. V. 1998. Nodular dermatofibrosis and cystic renal disease in three mixed-breed dogs and a Boxer dog. Vet. Dermatol. 9: $119-126$. 